Proyecciones Journal of Mathematics

Vol. 30, $\mathrm{N}^{o}$ 1, pp. 43-50, May 2011.

Universidad Católica del Norte

Antofagasta - Chile

\title{
On the instability of solutions of an eighth order nonlinear differential equation of retarded type
}

\author{
Cemil Tunç \\ Yüzüncü Yll University, Van Turkey \\ Received : August 2010. Accepted : April 2011
}

\begin{abstract}
In this paper, we give some sufficient conditions on the instability of the zero solution of a kind of eighth order nonlinear differential equations of retarded type by using the Lyapunov direct method. The obtained sufficient conditions improve an existing result in the literature.
\end{abstract}

Key words : Instability; the Lyapunov direct method; delay differential equation; eighth order.

AMS Classification numbers : 34 K20. 


\section{Introduction}

Since 1991 by now, the instability of solutions for various nonlinear scalar and vector differential equations of eighth order without delay has only been investigated by a few authors, see, Bereketoğlu [1], Tunç [6, 7], C. Tunç and E. Tunç [8] and the references contained therein. In addition, in 1996, Iyase [4] proved a result on the nonexistence of nontrivial periodic solutions to the nonlinear eighth order scalar differential equation without delay

$x^{(8)}+a_{1} x^{(7)}+a_{2} x^{(6)}+a_{3} x^{(5)}+a_{4} x^{(4)}+a_{5} x^{\prime \prime \prime}+f_{6}\left(x^{\prime}\right) x^{\prime \prime}+f_{7}(x) x^{\prime}+f_{8}(x)=0$.

Consider the linear constant coefficient differential equation of eighth order

$$
x^{(8)}+a_{1} x^{(7)}+a_{2} x^{(6)}+a_{3} x^{(5)}+a_{4} x^{(4)}+a_{5} x^{\prime \prime \prime}+a_{6} x^{\prime \prime}+a_{7} x^{\prime}+a_{8} x=0 .
$$

It is well known that the auxiliary equation of Eq. (1.2) is given by

$$
\psi(\lambda) \equiv \lambda^{8}+a_{1} \lambda^{7}+a_{2} \lambda^{6}+a_{3} \lambda^{5}+a_{4} \lambda^{4}+a_{5} \lambda^{3}+a_{6} \lambda^{2}+a_{7} \lambda+a_{8}=0 .
$$

If $\beta$ is an arbitrary real number, then the real part of $\psi(i \beta)$ is given by

$$
\phi(\beta)=\beta^{8}-a_{2} \beta^{6}+a_{4} \beta^{4}-a_{6} \beta^{2}+a_{8} .
$$

It is also well known that if

$$
a_{2} \leq 0, a_{4} \geq 0, a_{6} \leq 0, a_{8}>0
$$

in which case $\phi(\beta)>0$, then the auxiliary equation cannot have any purely imaginary root whatever. It therefore follows from general theory that Eq. (1.2) does not has a periodic solution except $x=0$. An analogous consideration of the imaginary part of $\psi(i \beta)$ also leads to conditions on $a_{1}$, $a_{3}, a_{5}$ and $a_{7}$ for the nonexistence of any periodic solution of Eq. (1.2) other than $x=0$. 
In this paper, instead of Eq. (1.1), we consider the eighth order nonlinear functional differential equation of delay type

$$
\begin{aligned}
& x^{(8)}+a_{1} x^{(7)}+a_{2} x^{(6)}+a_{3} x^{(5)}+a_{4} x^{(4)}+a_{5} x^{\prime \prime \prime}+f_{6}\left(x^{\prime}\right) x^{\prime \prime}+f_{7}(x) x^{\prime} \\
& \left(1.3 \ngtr f_{8}\left(x, x(t-r), x^{\prime}, x^{\prime}(t-r), \ldots, x^{(7)}, x^{(7)}(t-r)\right)=0 .\right.
\end{aligned}
$$

We write Eq. (1.4) in system form as

$$
\begin{gathered}
x_{1}^{\prime}=x_{2}, x_{2}^{\prime}=x_{3}, x_{3}^{\prime}=x_{4}, x_{4}^{\prime}=x_{5}, x_{5}^{\prime}=x_{6}, x_{6}^{\prime}=x_{7}, x_{7}^{\prime}=x_{8}, \\
x_{8}^{\prime}=-a_{1} x_{8}-a_{2} x_{7}-a_{3} x_{6}-a_{4} x_{5}-a_{5} x_{4}-f_{6}\left(x_{2}\right) x_{3}-f_{7}\left(x_{1}\right) x_{2} \\
(1.4)-f_{8}\left(x_{1}, x_{1}(t-r), x_{2}, x_{2}(t-r), \ldots, x_{8}, x_{8}(t-r)\right),
\end{gathered}
$$

which was obtained as usual by setting $x=x_{1}, x^{\prime}=x_{2}, x^{\prime \prime}=x_{3}, x^{\prime \prime \prime}=x_{4}$, $x^{(4)}=x_{5}, x^{(5)}=x_{6}, x^{(6)}=x_{7}$ and $x^{(7)}=x_{8}$ in (1.3), where $r$ is a positive constant, $a_{1}, a_{2}, a_{3}, a_{4}$ and $a_{5}$ are some constants, the primes in Eq. (1.3) denote differentiation with respect to $t, t \in \Re_{+}, \Re_{+}=[0, \infty)$; $f_{6}, f_{7}$ and $f_{8}$ are continuous functions on $\Re, \Re, \Re^{16}$, respectively, with $f_{8}\left(0, x_{1}(t-r), \ldots, x_{8}(t-r)\right)=0$, and satisfy a Lipschitz condition in their respective arguments. Hence, the existence and uniqueness of the solutions of Eq. (1.3) are guaranteed (see Èl'sgol'ts [2, pp.14, 15]). We assume in what follows that $x_{1}(t), x_{2}(t), x_{3}(t), x_{4}(t), x_{5}(t), x_{6}(t), x_{7}(t)$ and $x_{8}(t)$ are abbreviated as $x_{1}, x_{2}, x_{3}, x_{4}, x_{5}, x_{6} x_{7}$ and $x_{8}$, respectively.

The aim of this paper is to improve the result established in [4, Theorem 1] to the eighth order nonlinear delay differential equation (1.3) for the instability of its zero solution. Our motivation comes from the papers [1, $3,4,5,6,7,8,9]$.

As known, in general, it is not possible to solve all linear and nonlinear differential equations, except numerically. Therefore, it is very important obtaining information for the qualitative behaviors of solutions when there is no analytical expression for solutions of any differential equation under investigation. It should be noted that the Lyapunov's second (or direct) method is the most effective tool to discuss the instability of solutions of nonlinear differential equations of higher order, when there is no analytical expression for solutions. The technique is also called the direct method, because it can be applied directly to the differential equation under study, without any knowledge of solutions, provided the person using the method is clever to construct or define an appropriate the right auxiliary function, the Lyapunov function or functional. Here, by means of the Lyapunov direct method, we prove a theorem for the zero solution of Eq. (1.3) to 
be unstable. That is to say that, by defining a Lyapunov function, we will accomplish the result to be established in this work.

Let $r \geq 0$ be given, and let $C=C\left([-r, 0], \Re^{n}\right)$ with

$$
\|\phi\|=-r \leq s \leq 0 \max |\phi(s)|, \quad \phi \in C .
$$

For $H>0$ define $C_{H} \subset C$ by

$$
C_{H}=\{\phi \in C:\|\phi\|<H\} .
$$

If $x:[-r, a] \rightarrow \Re^{n}$ is continuous, $0<A \leq \infty$, then, for each $t$ in $[0, A)$, $x_{t}$ in $C$ is defined by

$$
x_{t}(s)=x(t+s),-r \leq s \leq 0, t \geq 0 .
$$

Let $G$ be an open subset of $C$ and consider the general autonomous differential system with finite delay

$$
\dot{x}=F\left(x_{t}\right), x_{t}=x(t+\theta),-r \leq \theta \leq 0, t \geq 0,
$$

where $F: G \rightarrow \Re^{n}$ is continuous and maps closed and bounded sets into bounded sets. It follows from these conditions on $F$ that each initial value problem

$$
\dot{x}=F\left(x_{t}\right), x_{0}=\phi \in G
$$

has a unique solution defined on some interval $[0, A), 0<A \leq \infty$. This solution will be denoted by $x(\phi)($.$) so that x_{0}(\phi)=\phi$.

Definition. The zero solution, $x=0$, of $\dot{x}=F\left(x_{t}\right)$ is stable if for each $\varepsilon>0$ there exists $\delta=\delta(\varepsilon)>0$ such that $\|\phi\|<\delta$ implies that $|x(\phi)(t)|<\varepsilon$ for all $t \geq 0$. The zero solution is said to be unstable if it is not stable.

Theorem A. Suppose there exists a Lyapunov function $V: G \rightarrow \Re_{+}$such that $V(0)=0$ and $V(x)>0$ if $x \neq 0$. If either

(i) $\dot{V}(\phi)>0$ for all $\phi$ in $G$ for which

$$
V[\phi(0)]=-s \leq t \leq 0 \max V[\phi(s)]>0
$$

or

(ii) $\dot{V}(\phi)>0$ for all $\phi$ in $G$ for which

$$
V[\phi(0)]=-s \leq t \leq 0 \min V[\phi(s)]>0,
$$

then $x=0$ of $\dot{x}=F\left(x_{t}\right)$ is unstable (see Haddock and Ko [3]). 


\section{The main results}

The main result of this paper is given by the following theorem.

Theorem. Together with all the assumptions imposed to the functions $f_{6}$, $f_{7}$ and $f_{8}$ in Eq. (1.3), we assume that there exist constants $a_{1}, a_{2}$ and $a_{4}$ such that the conditions

$$
\begin{gathered}
a_{1}>0, a_{2}<0, a_{4}>0, f_{6}\left(x_{2}\right) \leq 0 \\
x_{1} f_{8}\left(x_{1}, \ldots, x_{8}(t-r)\right)>0,\left(x_{1} \neq 0\right),
\end{gathered}
$$

hold for arbitrary $x_{1}, x_{1}(t-r), \ldots, x_{8}(t-r)$. Then, the zero solution, $x=0$, of Eq. (1.3) is unstable.

Remark. Note that there is not any restriction on the function $f_{7}$. Next, the proof of the above theorem is based on the instability criteria of Krasovskii [5]. According to these criteria, it is necessary to show here that there exists a Lyapunov function $V \equiv V\left(x_{1}, x_{2}, \ldots, x_{8}\right)$ which has the following three properties, Krasovskii properties, say $\left(K_{1}\right),\left(K_{2}\right)$ and $\left(K_{3}\right)$ :

$\left(K_{1}\right)$ In every neighborhood of $(0,0,0,0,0,0,0,0)$, there exists a point $\left(\xi_{1}, \xi_{2}, \ldots, \xi_{8}\right)$ such that $V\left(\xi_{1}, \xi_{2}, \ldots, \xi_{8}\right)>0$,

$\left(K_{2}\right)$ the time derivative $\dot{V} \equiv \frac{d}{d t}\left(x_{1}, x_{2}, \ldots, x_{8}\right)$ along solution paths of (1.4) is positive semi-definite,

$\left(K_{3}\right)$ the only solution $\left(x_{1}, x_{2}, \ldots, x_{8}\right)=\left(x_{1}(t), x_{2}(t), \ldots, x_{8}(t)\right)$ of $(1.4)$ which satisfies

$\frac{d}{d t} V\left(x_{1}, x_{2}, \ldots, x_{8}\right)=0,(t \geq 0)$, is the trivial solution $(0,0,0,0,0,0,0,0)$.

Proof. Consider the function $V=V\left(x_{1}, x_{2}, \ldots, x_{8}\right)$ defined by

$$
\begin{aligned}
V & =-x_{1} x_{8}-a_{1} x_{1} x_{7}-a_{2} x_{1} x_{6}-a_{3} x_{1} x_{5}-a_{4} x_{1} x_{4}-a_{5} x_{1} x_{3} \\
& +x_{2} x_{7}+a_{1} x_{2} x_{6}+a_{2} x_{2} x_{5}+a_{3} x_{2} x_{4}+a_{4} x_{2} x_{3} \\
& +\frac{1}{2} a_{5} x_{2}^{2}-x_{3} x_{6}-a_{1} x_{3} x_{5}-a_{2} x_{3} x_{4}-\frac{1}{2} a_{3} x_{3}^{2} \\
& +x_{4} x_{5}+\frac{1}{2} a_{1} x_{4}^{2}-x_{1} \int_{0}^{x_{2}} f_{6}(s) d s-\int_{0}^{x_{1}} f_{7}(s) s d s .
\end{aligned}
$$

It follows that

$$
V(0,0,0,0,0,0,0,0)=0
$$

and

$$
V(0,0,0, \varepsilon, 0,0,0,0)=\frac{1}{2} a_{1} \varepsilon^{2}>0
$$


for all sufficiently small $\varepsilon$ so that every neighborhood of the origin in the $\left(x_{1}, x_{2}, \ldots, x_{8}\right)-$ space contains points $\left(\xi_{1}, \xi_{2}, \ldots, \xi_{8}\right)$ such that $V\left(\xi_{1}, \xi_{2}, \ldots, \xi_{8}\right)>$ 0 , which verifies the property $\left(K_{1}\right)$ of Krasovskii [5].

Let

$$
\left(x_{1}, x_{2}, \ldots, x_{8}\right)=\left(x_{1}(t), x_{2}(t), \ldots, x_{8}(t)\right)
$$

be an arbitrary solution of (1.4).

Along solutions of the system (1.4), we obtain the time derivative of the function $V$ in (2.1) as the following:

$$
\dot{V} \equiv \frac{d V}{d t}=x_{5}^{2}-a_{2} x_{4}^{2}+a_{4} x_{3}^{2}+f_{8}\left(x_{1}, \ldots, x_{8}(t-r)\right) x_{1}-x_{2} \int_{0}^{x_{2}} f_{6}(s) d s .
$$

In view of the assumptions $a_{2}<0, a_{4}>0, f_{6}\left(x_{2}\right) \leq 0$ and $x_{1} f_{8}()>$. $0,\left(x_{1} \neq 0\right)$, it follows that

$$
\dot{V}=x_{5}^{2}-a_{2} x_{4}^{2}+a_{4} x_{3}^{2}+f_{8}\left(x_{1},, \ldots, x_{8}(t-r)\right) x_{1}-x_{2} \int_{0}^{x_{2}} f_{6}(s) d s>0 .
$$

Thus, if the assumptions of the theorem hold then $\dot{V}$ is positive semi definite, which verifies the property $\left(K_{2}\right)$ of Krasovskii [5].

On the other hand, $\dot{V}=0$ for all $t \geq 0$ necessarily implies that $x_{1}=0$ and therefore also that

$$
\begin{gathered}
x_{1}=x=0, x_{2}=x^{\prime}=0, x_{3}=x^{\prime \prime}=0, x_{4}=x^{\prime \prime \prime}=0, x_{5}=x^{(4)}=0, \\
x_{6}=x^{(5)}=0, x_{7}=x^{(6)}=0, x_{8}=x^{(7)}=0
\end{gathered}
$$

for all $t \geq 0$. Hence,

$$
x_{1}=x_{2}=x_{3}=x_{4}=x_{5}=x_{6}=x_{7}=x_{8}=0,(t \geq 0) .
$$

Furthermore, in view of $\frac{d}{d t} V\left(x_{1}, x_{2}, \ldots, x_{8}\right)=0$ and the system (1.4), we can also easily obtain $x_{1}=x_{2}=x_{3}=x_{4}=x_{5}=x_{6}=x_{7}=x_{8}=0$ since $f_{8}\left(\xi_{1}, \ldots, \xi_{8}(t-r)\right)=0$ if and only if $\xi_{1}=0$, which verifies the property $\left(K_{3}\right)$ of Krasovskii [5]. It now follows that the Lyapunov function $V$ thus has all the requisite Krasovskii properties subject to the conditions in the theorem. By the above discussion, we conclude that the zero solution of Eq. (1.3) is unstable. The proof of the theorem is completed. 


\section{References}

[1] Bereketoğlu, H., On the instability of trivial solutions of a class of eighth-order differential equations. Indian J. Pure Appl. Math. 22, No. 3, pp. 199-202, (1991).

[2] Èl'sgol'ts, L. È., Introduction to the theory of differential equations with deviating arguments. Translated from the Russian by Robert J. McLaughlin Holden-Day, Inc., San Francisco, Calif.-LondonAmsterdam, (1966).

[3] Haddock, John R. ; Ko, Y., Liapunov-Razumikhin functions and an instability theorem for autonomous functional-differential equations with finite delay. Second Geoffrey J. Butler Memorial Conference in Differential Equations and Mathematical Biology (Edmonton, AB, 1992). Rocky Mountain J. Math. 25, No. 1, pp. 261-267, (1995).

[4] Iyase, S. A., Periodic solutions of certain eighth order differential equations. J. Nigerian Math. Soc. 14/15, pp. 31-39, (1995/1996).

[5] Krasovskii, N. N., On conditions of inversion of A. M. Lyapunov's theorems on instability for stationary systems of differential equations. (Russian) Dokl. Akad. Nauk SSSR (N.S.) 101, pp. 17-20, (1955).

[6] Tunç, C., Instability of solutions of a certain non-autonomous vector differential equation of eighth-order. Ann. Differential Equations 22, No. 1, pp. 7-12, (2006).

[7] Tunç, C., Nonexistence of nontrivial periodic solutions to a class of nonlinear differential equations of eighth order. Bull. Malays. Math. Sci. Soc. (2) 32, No. 3, pp. 307-311, (2009).

[8] Tunc, C.; Tunc, E., An instability theorem for a class of eighth-order differential equations. (Russian) Differ. Uravn. 42 (2006), No. 1, 135138, 143; translation in Differ. Equ. 42, no. 1, pp. 150-154, (2006).

[9] Tunç, C., An instability theorem for solutions of a kind of eighth order nonlinear delay differential equations. World Applied Sciences Journal 12 (5) : pp. 619-623, (2011). 


\section{Cemil Tunç}

Department of Mathematics,

Faculty of Arts and Sciences,

Yüzüncü Yıl University,

65080, VAN-TURKEY

e-mail : cemtunc@yahoo.com 